\title{
Civilisations
}

Revue internationale d'anthropologie et de sciences

humaines

$48 \mid 2001$

La question de l'islam et de l'Etat à l'aube du XXIe siècle

\section{Note sur la séparation du religieux et du pouvoir dans la pensée politique musulmane}

\section{Olivier Carré}

\section{(2) OpenEdition}

\section{Journals}

Édition électronique

URL : http://journals.openedition.org/civilisations/3454

DOI : 10.4000/civilisations.3454

ISSN : 2032-0442

Éditeur

Institut de sociologie de l'Université Libre de Bruxelles

\section{Édition imprimée}

Date de publication : 31 août 2001

Pagination : 15-26

ISSN : 0009-8140

\section{Référence électronique}

Olivier Carré, « Note sur la séparation du religieux et du pouvoir dans la pensée politique musulmane ». Civilisations [En ligne], 48 | 2001, mis en ligne le 01 août 2004, consulté le 30 avril 2019. URL : http:// journals.openedition.org/civilisations/3454 ; DOI : 10.4000/civilisations.3454 


\title{
NOTE SUR LA SÉPARATION DU RELIGIEUX ET DU POUVOIR DANS LA PENSÉE POLITIQUE MUSULMANE
}

\author{
Olivier CARRÉ
}

\section{INTRODUCTION}

Dans cette note, relue et modifiée après les débats de notre Colloque, j'entends corriger une idée faussée de l'islam, dangereuse pour les minorités musulmanes en Europe. En voici une expression nette (R. Rémond, 1999) : «(Avec l'islam) on a une religion qui ne comporte pas, pour le moment, apparemment, en elle de possibilité d'interface, pour ainsi dire, pour la laïcité, puisqu'il y a, non pas confusion mais fusion totale entre la communauté religieuse et la société civile. Le Coran est à la fois le code civil et le code pénal; par conséquent c'est au Coran qu'on trouve l'inspiration du gouvernement. Il n'y a pas de distinction, dans le cas des États musulmans, entre le chef de l'État et le chef religieux. Alors, cet islam coutumier, à qui la notion de laïcité ou de séparation est étrangère, comment l'État français peut lui étendre le bénéfice de la liberté religieuse, qui, en soi, ne pose pas de question ?» Les mots soulignés par moi désignent précisément les notions fausses ou faussées qui identifient peu ou prou l'islam à l' «islamisme» (selon le mot reçu). Voyons pourquoi, conformément au vœu souvent exprimé au cours de notre colloque, une semblable assimilation de l'islam à l'islamisme est foncièrement injustifiée, même si le discours de l'appareil actuel de l'islam va assez dans ce sens.

En adoptant une distinction féconde à propos de la tradition (R.J. Campiche, 1995), je rappellerai quelle est la «tradition longue» ou «grande tradition» de la pensée politique musulmane, puis en quoi la pensée dominante actuelle relève plutôt d'une «tradition courte» ou «petite tradition», troisièmement quelle est la doctrine des groupes extrémistes actuels, enfin, last but not least, les forces intellectuelles de résistance et véritable renaissance islamique anti- et post-«islamiste». 


\section{LA GRANDE TRADITION DE LA PENSÉE POLITIQUE MUSULMANE}

C'est la doctrine élaborée et systématisée bien après les événements tragiques de la succession du Prophète et de la «grande discorde» (H. Djait, 1989) entre shiites, sunnites et khârijites. Elle prend une position nette (A.K. Lambton, 1981) contre les théories totalitaires telle celle de Farâbi qui islamise la République de Platon, et tel l'art politique pratique des traités d'Ibn Muqaffa, héritier du despotisme perse. Aussi cette pensée politique musulmane est-elle caractérisée comme un «quiétisme politique» (A.K. Lambton, 1981 : passim; B. Lewis, 1988 : passim; P.J. Vatikiotis, 1992 (1987) : 99, 33sq.), autant en sunnisme qu'en shiisme imâmite.

En tradition shiite imâmite, en effet, la taqiya (dissimulation, restriction mentale), dont le quiétisme politique est un élément central, est rendue obligatoire à la fin du $\mathrm{X}^{\mathrm{e}}$ siècle, pour toute la durée du temps de l'Occultation du douzième Imâm, c'est-à-dire jusqu'aux prodromes du Jour du Jugement (S.A. Arjomand, 1984). La grande tradition sunnite insiste, elle aussi, sur le caractère non théocratique du pouvoir après le Prophète et les «khalifes bien dirigés» qui, eux aussi, mais eux seuls, se sont conférés les deux pouvoirs du Prophète, et politique et religieux, ce qui, précisément, n'allait pas de soi (P. Crone, M. Hinds, 1986).

Il y a donc, dans cette théorie systématisée depuis le $\mathrm{XI}^{\mathrm{e}}$ siècle (contrairement à ce qu'affirme certaine tradition orientaliste persistante) et non seulement dans la pratique, une séparation du religieux et du politique, en sunnisme comme en shiisme duodécimain (N. Ayubi, 1991; O. Carré, 1993a), en distinguant entre la prégnance forte du religieux sur la vie sociale et donc le droit d'une part et la séparation du religieux et du politique d'autre part. Certes, la séparation est presque abolie au début du règne Safavide en Iran décrété shiite dans les années 1500 et, de nouveau, dans les années 1980 avec Khomeiny qui avait précédemment, en tant qu'ayatollah, aboli doctrinalement la taqiya et le quiétisme politique qui en découle. En sunnisme aussi, la séparation est suspendue lors des brefs «mahdismes» (selon le vocabulaire de Max Weber), récurrents dans l'histoire musulmane jusqu'au Mahdi du Soudan, au mouvement sénoussi et à Abdelkrim, notamment. Pour être plus pertinent, parlons de distinction nette plutôt que de séparation au sens strict, en islam comme en christianisme et en beaucoup de traditions religieuses ou simplement «humanistes», qui entendent toutes, bien évidemment, que le pouvoir politique soit contrôlé par leurs exigences de justice. Le musulman M. Imara, 1978 et le catholique J.-Y. Calvez, 1985, par exemple, qui tous deux reflètent une réflexion religieuse éclairée mais traditionnelle sur le politique, s'expriment rigoureusement dans les mêmes termes : distinction totale, pas séparation absolue. C'est cela que l'on entend couramment par «séparation» institutionnelle du religieux et du politique, qui ne signifie nullement l'apolitisme des croyants et des «Églises».

En politique musulmane de grande tradition, selon la synthèse d'Ibn Khaldûn (m.1406), qui n'innove nullement ni en théologie ni en fiqh (droit et morale pratique) ni en politique (G. Anawati, L. Gardet, 1948), Dieu n'intervient plus immédiatement et directement comme au temps miraculeux, révolu et non réitérable, de la théocratie par la bouche et la geste du Prophète et, à un moindre degré, de ses premiers successeurs, «khalifes bien dirigés» en sunnisme, ou «Imâms infaillibles» inspirés en shiisme (O. Carré, 1991 : 22sq, 33sq, 42sq). Telle est la tradition bien établie de la séparation, non seulement effective mais aussi doctrinale, entre politique et religieux, lē politique n'ayant rien de religieux ni de «révélé» dans sa substance. En particulier, cette tradition longue récuse nettement le jihâd guerrier interne contre des chefs politiques injustes ou contre les apostats détenant une autorité politique ou intellectuelle et morale, ainsi qu'elle condamne l'anathème, takfir , qui déclare apostats et passibles de mort 
tel et tel. De telles pratiques, en bonne tradition, relèvent de l'associationisme ou rejet de l'unicité absolue de Dieu, du fait que celui qui porte la rébellion violente ou l'anathème s'associe à Dieu l'Omniscient et le Juge en prétendant scruter les cœurs et les juger (H. Laoust, 1977 (1965) : Conclusion).

\section{LA TRADITION COURTE}

Malheureusement, c'est une «tradition courte» en pensée politique, non plus la grande tradition sunnite ou shiite, qui a cours de nos jours dans les cercles autorisés de l'islam. Dans ce néo-hanbalisme en rupture avec le pragmatisme politique accentué du hanbalisme, la variante wahhâbite joue un rôle croissant depuis au moins un siècle, même si elle est, comme «secte», limitée à l'Arabie Saoudite et au Qatar, avec aussi quelques influences anciennes au Maroc et dans le continent indien, et, tout récemment, au Caucase musulman. C'est Ibn Taymiyya, au début du XIVe siècle et, plus encore, ses disciples du XVe siècle (Ibn Qayyim alJawziyya notamment) qui innovent en instaurant un «néo-hanbalisme» qui se ressource à la primitive tendance khârijite extrême ou, plus tard, shiite ismaillienne extrémiste, qui prône et applique avec violence la fusion des deux pouvoirs. Même ce qu'on a nommé le «néo-islam» réformiste de la seconde moitié du XIX et début du $\mathrm{XX}^{\mathrm{e}}$ siècles, pénétré sans doute des «Lumières» européennes (A. Laroui, 1987), est marqué parce courant intégraliste, contrairement à ce que l'on croit souvent : la traditionnelle séparation du spirituel et du pouvoir, perçue comme une passivité servile de l'autorité religieuse, est en effet rejetée par les «réformistes (C. Mansour, 1975), du moins dans la ligne de Ridâ et à l'exception de Abduh, comme me le rappelle Robert Anciaux en ce colloque; Abduh en effet a nettement exprimé la séparation, en particulier lors de l'élaboration de la Constitution égyptienne de 1898, séculière puisqu'elle évite même d'indiquer l'islam comme religion de l'État, non-confessionnalité reprise dans la Constitution de 1923 mais, conformément au projet de Constitution islamique des Frères musulmans en 1952, abolie depuis celle de Nasser en 1957 comme dans les autres pays arabes actuels.

Aujourd'hui, s'il y a, certes, les «islamistes» d'un côté et l'islam, traditionaliste ou moderniste, de «l'appareil» mondial de l'autre, les instances de ce dernier s'alignent souvent sur des positions «islamistes». Je parle de l'Organisation de la Conférence Islamique (OCI), de la Ligue islamique mondiale surtout (d'inspiration saoudienne) avec ses différents organismes, du Congrès du monde musulman également (d'inspiration pakistanaise), des congrès des uléma réunis au Caire par al-Azhar, et des différentes réunions d'uléma ou d'autres autorités intellectuelles musulmanes dans certaines capitales musulmanes. L'influence des Frères musulmans égyptiens, palestiniens et syriens y est et reste très sensible depuis les années 1950 dans les congrès musulmans et les réunions du Congrès du monde musulman, puis dans celles des instances, plus récentes, de la Ligue islamique (R. Schulze, 1990 : 108-109).

Leurs documents importants assez récents sur la question sont la Constitution islamique du congrès des uléma au Caire (1979), la Déclaration islamique universelle des droits de l'homme du Conseil islamique européen à Paris (1981 \& 1983: traductions anglaise et française rigoureuses annotées, sensiblement différentes des traductions officielles; \& A.E. Mayer 1991, livre consacré à cette Déclaration et, en même temps, à la condamnation et exécution de M. Tahâ au Soudan), et Modèle de Constitution islamique du Congrès du monde musulman à Islamabad (1983). Ils insistent tous les trois, comme les Frères musulmans, sur l'absolue nécessité de «restaurer la sharia», définie, en annexe de la Déclaration..., comme tout ce qui découle des quelques versets juridiques du Coran (qui ont l'autorité directe de Dieu) et des innombrables hadiths (traditions écrites ayant l'autorité du Prophète) juridiques ou éthiques, 
avec un effort déjà ancien d'élaboration moderne. Ils reconnaissent aussi les droits individuels, dont le droit légitime à la rébellion contre un pouvoir musulman oppressif, revendication très ibn-taymiyyenne. Mais surtout, ces expressions solennelles et autorisées de l'orthodoxie actuelle affirment la fusion essentielle du religieux et du politique en islam : «Dîn=Dunya=Dawla, DDD», devise fautive de la «raison islamique» régnante (M. Arkoun, 1984; 1986). Elles ne sont donc, hélas, pas substantiellement contraires aux doctrines des groupes extrémistes, qu'elles condamnent pourtant incidemment. Les voici.

\section{L'EXTRÉMISME ISLAMIQUE}

Nullement traditionnelle en islam tant sunnite que shiite imâmite, est exprimée, dans la mouvance sunnite, avec intelligence et passion, par l'Égyptien Sayyid Qutb pendu par Nasser en 1966 et par l'Indien, puis Pakistanais A.A. Mawdûdi (m.1979), et reprise et amplifiée avec prestige et influence par le Pakistanais Khurshid Ahmad, disciple de Mawdûdî. Dans la mouvance shiite, notons les Iraniens A. Shariati (très semblable à Qutb, un laïc lui aussi, sur beaucoup de points) et R. Khomeiny, et, avec originalité et intelligence, le Libanais Muhammad Husayn Fadlallâh (O. Carré, 1991 :248-256). Il s' agit là d'un courant devenu bruyant parce que souvent dangereux qui, même dans ses élaborations théologico-politiques raffinées qu'on a qualifiées de «théologie de la libération» islamique (S. Akhtar, 1991), n'a plus grand chose à voir, en pensée politique, avec la grande tradition musulmane.

En effet, l'utopie d'une théocratie directe, non hiérocratique certes, puisque sans hiérarchie religieuse au sens prégnant, mais aux ordres d'un "petit groupe de croyants sincères» selon l'expression de Qutb (O. Carré, 1984: passim), suit le courant d'un «néo-hanbalisme» (E. Sivan, 1985; O. Carré, 1993a) en politique. Héritée d'Ibn Taymiyya et de ses disciples, la théorie du pouvoir et de la guerre dans cette «tradition courte» dévie de la «grande tradition» ou «tradition longue» de la pensée musulmane. Les groupes extrémistes, à l'encontre de la grande tradition, entendent et pratiquent le jihâd comme obligation individuelle, et non seulement collective (étatique), contre un environnement à la fois interne et mondial de néopaganisme ou ignorance anté-islamique. Ils pratiquent l'anathème (takfir) contre les apostats qui exercent un pouvoir, en particulier les hommes d'État estimés injustes, car les doctrines extrémistes, depuis l'origine des conflits dans l'Islam, confondent faute morale et incroyance, à l'encontre de la grande tradition. L'anathème, en droit musulman, entraîne la mise à mort sans procès car il désigne des personnes en état de guerre contre Dieu. Certains extrémistes vont jusqu'à prôner et pratiquer, comme les Ismaïliens extrêmes du Moyen Âge, l'assassinat suicidaire ciblé comme acte religieux suprême. L'objectif est d'instaurer immédiatement, par la technique du coup d'État, le pouvoir exclusif de Dieu et ce qu'ils estiment être sa Loi (sharia).

C'est ainsi que les Frères musulmans prennent le pouvoir au Soudan en 1989, comme un groupe de religieux le fait en Iran en 1981 au terme d'un mouvement révolutionnaire de grande ampleur, et, comme les autorités iraniennes depuis lors, ils estiment avoir réalisé peu à peu une codification moderne et d'avenir de la sharia. D'une manière générale, «l'application de la sharia», dans cette mouvance, même dans l'aile modérée des Frères musulmans historiques, consiste pour l'essentiel en ceci :

1. des lois pénales ou, parfois, le «prix du sang» en substitution, peines mentionnées dans le Coran et (surtout) dans les innombrables «Traditions» (hadiths) : peine capitale ou de mutilation contre les apostats (la conversion d'une personne musulmane à une autre foi ou à l'athéisme ou théisme sans religion instituée étant civilement prohibée), contre les rebelles à l'ordre public musulman, contre les fornicateurs (dont les adultères) et les diffamateurs en cette 
matière, contre le grand banditisme, lois édictées sans élaboration juridique moderne sérieuse et un faible sens de l'histoire des sociétés et du droit;

2. un droit civil comportant un code de la famille qui soumet la femme musulmane à une tutelle permanente et prohibe pour elle tout mariage interconfessionnel, et un code du statut personnel discriminant les non musulmans et leur imposant une capitation à la place ou, parfois, en plus des taxes des seuls musulmans;

3. une loi constitutionnelle instaurant un Conseil consultatif ou shûra, sans pouvoir décisif auprès du Prince ou Imâm;

4. des lois économiques prohibant en principe non pas l'usure seulement, mais, à l'encontre de Abduh une fois encore, tout intérêt sur l'argent, et imposant la taxe coranique sur tous les revenus et les capitaux, qui est l'un des cinq piliers de l'islam. La justice sociale, si sensible à Qutb dans sa Social Justice in Islam, est de nos jours en général laissée à l'arrièreplan, et l'on ne met en avant que les lois pénales et civiles supposées coraniques («l'application de la sharia» est la devise) et aussi, parfois, pour y parvenir, un jihâd interne contre les pouvoirs établis. Ces derniers sont, certes, presque tous autoritaires et policiers ou militaires, sans espace réel à la lutte purement politique; toutefois la démocratie islamique locale prônée par la doctrine de la shûra selon Qutb est elle aussi laissée en sourdine dans ces États «qutbistes», qui se veulent et sont eux aussi très autoritaires à tendance totalitaire.

Attardons-nous un moment sur l'aspect assez peu orthodoxe de la doctrine du jihâd qui inspire toute l'action de cette tendance à travers le monde musulman. Un intervenant au colloque, très informé de la littérature «islamiste» diffusée en Europe, a souligné la référence continuelle à l'autorité de Ibn Taymiyya, tandis qu'un autre intervenant, à propos du Hamas en Palestine, déniait a priori l'influence d'auteurs médiévaux comme Ibn Taymiyya et ses disciples, contrairement à ce qui a été soigneusement étudié par E. Sivan, 1985 (1992). Cette influence est en effet centrale et conséquente, comme je vais le montrer. En grande tradition, la guerre-pour-Dieu, jihâd, est une obligation, mais sans «contrainte en religion», restriction remarquable dans le contexte d'alors, qui sera la base du futur «maintien et respect des autres religions monothéistes dans la société islamique» établie (R. Blachere, 1950:806, note sur Coran 2.257). Certes, à l'origine de l'islam institué, on reconnaît le «légitime impérialisme de l'islam», mais seulement lors des quatre premiers khalifes et eux seuls (Henri Laoust, 1939 : 217). Et s'il y a accord des docteurs sur la valeur abrogeante décisive (principe affirmé clairement par Coran 2, v.100) des versets les plus tardifs, qui sont les plus intransigeants, sur le devoir et les cibles du jihâd, il y a le même accord sur l'interprétation irénique de ces mêmes versets pour après l'époque prophétique et immédiatement postérieure, et toutes les écoles, «orthodoxes» ou non, s'accordent pour, après cette période primitive miraculeuse, un jihâd étatique soit pour protéger les frontières soit contre «les dissidents» seulement (A. Morabia, 1995). Cela représente le pacifisme fondamental des empires musulmans. Notamment l'État ottoman favorisa l'essor des non-musulmans dans les provinces arabes d'Orient (Courbage \& P. Fargues, 1992).

Mais Ibn Taymiyya (m. 1328), lui, prêche un jihâd étatique sunnite contre les sectes extrémistes issues du shiisme considérées comme renégates à l'islam, et que les shiites imâmites combattent aussi (H. Laoust, 1939 : 361 sq.). Sa position rompt avec la tradition particulièrement irénique et empirique de son école juridique hanbalite, et se rapproche de la position shiite imâmite idéale et de celle des Khârijites. Le jihâd y est le sixième «pilier» de l'islam, contrairement à la grande tradition sunnite qui n'en a que cinq, mais les shiites imâmites 
en interdisent l'exercice effectif durant l'Occultation, ce qui les ramène en théorie comme en pratique à la position sunnite. D'autre part, seuls, dans l'islam médiéval, ces courants nonorthodoxes partagent la vue d'un jihâd entre musulmans de par l'obligation de rébellion violente contre les dirigeants injustes (donc incroyants selon eux) et, dans cette ligne, le sunnite Ibn Taymiyya prône la guerre étatique contre le pouvoir des Tatars parce qu'ils seraient des musulmans douteux. Le jihâd, il est vrai, comme guerre purificatrice de la société musulmane elle-même constitue bien un principe théorique chez les grands classiques, qui, toutefois, évitent tous de l'appliquer et, comme Ghazâli (m. 1111), préfèrent la résistance passive d'uléma orchestrant une opinion publique hostile aux mauvais gouvernants et mauvais décrets ou lois. Ibn Taymiyya et ses disciples prônent au contraire la pratique de révoltes violentes purificatrices. Enfin, Khârijites et Ismailiens prônent même, comme arme de guerre, le devoir religieux de «meurtre suicidaire» ou terrorisme martyre ciblé (A. Morabia, 1995, pour tout ce paragraphe). Ajoutons, au $\mathrm{XX}^{\mathrm{e}}$ siècle, la tendance assez pacifique de guerre exclusivement défensive de «l'école de Abduh», qui a largement imprégné la pensée musulmane actuelle et que rejette vivement Qutb et les «qutbistes».

Voilà assez précisément déterminés les aspects «hérétiques» de la doctrine des «extrémistes islamiques» actuels comme anciens en matière de tout ce qui touche à la guerre, notamment intestine. En particulier, les extrémistes des années 1980 réfèrent à Ibn Taymiyya pour justifier le même combat armé que lui contre les autorités politiques syriennes actuelles, qui appartiennent au shiisme extrémiste (alaouite, qu'Ibn Taymiyya rattache à tort à l'ismaïlisme et non au shiisme imâmite), et contre tout pouvoir «néo-tatar» d'aujourd'hui, entendons baassiste ou nassérien qu'ils considèrent renégat (G. Michaud, alias M. Seurat, in O. Carré \& G. Michaud, 1983 : 172-177). Aussi les censeurs religieux institutionnels des «qutbistes» actuels et de Qutb lui-même (souvent non nommé) leur reprochent-ils de s'éloigner de la grande tradition pour verser «dans l'orientalisme» qui, selon eux, veut faire de l'islam une religion de la violence, de l'intransigeance et de l'expansion militaire même après la disparition du Prophète et des premiers khalifes ou du douzième Imâm, à la manière des seuls extrémistes non-orthodoxes, voire non musulmans, de jadis (Alî Jâdd al-Haqq, 1982). Toutefois, dans d'autres occasions, comme à propos des guerres successives en Afghanistan, les mêmes autorités religieuses suivent la tendance «néo-hanbalite» qui leur est devenue presque naturelle en matière politique.

Voici quelques opérations «islamistes» récentes non guerrières, avec la caution au moins passive des autorités musulmanes agréées : la condamnation à mort de M. Tâhâ au Soudan en 1985 pour rébellion contre l'ordre étatique sur la base de son apostasie par le fait qu'il s' opposait au nouveau code pénal prétendument conforme à la sharia (M.M. Taha, 1987; A. an-Na'im, 1990; A.E. Mayer, 1991); la fameuse fatwa de Khomeiny en 1989, qui n'était pas une fatwa en réalité, mais un décret de l'autorité étatique, qui est le contraire d'une fatwa qui éclaire mais ne contraint jamais (M. Mozaffari, $1998: 16 \mathrm{sq}$.), pour la mise à mort sans procès du romancier britannique Salman Rushdie pour apostasie dans tel écrit du fait qu'il est né musulman; en Égypte, les censures dans les années 1960 puis des fatwas des années 1980/1990 contre le roman symbolique majeur de Naguib Mahfouz (1995 (1958)), littéralement Les fils de notre quartier, à ne pas confondre (comme il est arrivé sur ARTE dans l'émission Metropolis du 7.6.1997) avec Récits de notre quartier, œuvre récente mineure, et finalement contre sa personne même; les procès, en Égypte encore, depuis 1992, contre Nasr Abû Zayd pour apostasie (et donc rébellion à l'ordre public!) sur la base de ses écrits exégétiques et de droit islamique de haut niveau, et sa condamnation au divorce de son épouse musulmane. De telles opérations sont devenues courantes, en Égypte notamment, à la faveur de la démocratisation 
de la justice, au nom d'une réislamisation aveugle et inculte du droit et des mours par un groupe de magistrats et avocats. On voit, hélas, dans ces événements une certaine connivence entre les islamistes et l'appareil de l'islam.

4. La tâche de résistance et de renaissance véritable contre cet obscurantisme religieux régnant aujourd'hui, résistance déjà ancienne, en Égypte notamment, reste donc immense et, on le voit, elle s'avère plus périlleuse qu'auparavant. Elle est pourtant urgente, car la perpétuation de l'épisode «islamiste» serait suicidaire pour l'Islam et sa civilisation, bien plus qu'elle ne met et ne mettrait en danger l'Occident. Le «terrorisme» des extrémistes islamiques, en effet, s'il endommage de temps à autre des personnes et des biens de l'Occident, est dévastateur des biens et des personnes, à assez grande échelle parfois, dans les pays musulmans : il détruit avant tout des musulmans et l'islam lui-même, L'islamisme contre l'islam (M.S. AlAshmawy, 1989 (1987)). La paresse de l'appareil actuel, si elle persévérait, contribuerait ainsi au suicide de l'islam et de sa civilisation. Le mérite de l' «islam parallèle» politique et violent, très minoritaire mais convaincu et bien organisé, avec un dynamisme durable surprenant (qui inclut le martyre), c'est qu'il pousse toutes les sociétés musulmanes à une inévitable réislamisation moderne. C'est plutôt hors «appareil» que se développe une pensée politique musulmane rénovée anti «islamiste», anti-«qutbiste», qui s'imposera peut-être peu à peu au sein même de l'islam de l'appareil, en accord avec la «tradition longue» au sens, bien sûr, de tradition vivante, novatrice.

Les écrits de Nasr Abû Zayd (cf.1999 (1993)) représentent une tâche remarquable en ce sens. Attardons-nous sur deux arguments majeurs produits par la mouvance «islamiste» ou par l'appareil islamique égyptien contre lui. Primo (A. Sinno, 1995 :4), il ose critiquer Sayyid Qutb ainsi que des «autorités reconnues» de l'Azhar et, refusant de tout ramener à la souveraineté exclusive de Dieu, il prône la sécularisation, qu'il prétend détacher de l'athéisme. On voit ainsi comment Qutb, vivement critiqué par les autorités religieuses musulmanes égyptiennes en 1965-1966 et par les Frères musulmans eux-mêmes à partir de 1969, est aujourd'hui intégré parmi les garants de «l'orthodoxie» de «l'appareil» actuel de l'islam. Et pour les nouveaux censeurs, devenus «qutbiens» donc, la sécularisation n'est entendue, comme chez Qutb, que comme un laïcisme athée anti-religieux ou irréligieux militant, au sens d'une exclusion ou séparation totale entre le politique et le droit d'une part et la croyance et les pratiques d'autre part, non d'une distinction et d'une neutralité non confessionnelle. L'autre reproche fondamental concerne sa méthode exégétique, par exemple de la part de la cheville ouvrière des procès de moralité islamique intentés en Égypte depuis 1993 : «Comment Nasr Abû-Zayd a-t-il pu écrire que le Coran n'est pas la Parole de Dieu? Comme il n'est pas juif ni chrétien, mais musulman, il est donc un apostat, qui a renié la religion musulmane dans sa totalité. Apostat, il doit être tué. Mais nous ne combattons pour autant pas la liberté de pensée» (Cheikh Yûsûf al-Badrî, 1997). Toute recherche sérieuse d'exégèse et d'herméneutique du Coran passe donc pour apostasie, et c'est pourtant le fondement de tout renouveau de la pensée religieuse musulmane, en particulier concernant le droit.

La sharia, dans cette pensée de renouveau, pas plus que dans la tradition longue, n'est un ensemble de codes, pénal et civil notamment, édictés par Dieu, mais une inspiration générale et l'expression sacralisée des grands principes, des fins générales du droit (A. Azmeh, 1993). Pas plus que le politique, le droit (hors les règles de la pratique religieuse) n'est substantiellement religieux ni «révélé», mais humain, naturel, même s'il est aussi révélé par une parole divine dans ses principes et dans quelques dispositions précises de droit civil (mariage, statut confessionnel) et de droit pénal qui ne sont intangibles que dans leurs principes. La pensée 
musulmane moderne éclairée, dans le sillage, notamment, de Fazlur Rahman (en Inde), de Khereddine (en Tunisie \& État ottoman) et de Ali Abdel-Razeq, Tâhâ Husein, Amin al-Khûli (en Égypte) et de plusieurs autres, dont, de nos jours, pêle-mêle et parmi bien d'autres, A. Khalafallâh, M.S. al-Ashmâwi, N.Abû Zayd en Égypte, M. Arkoun en France, M.A. alJâbiri au Maroc, M. Talbi en Tunisie, Nurcholis Madjid en Indonésie, contribue, avec le sens de l'urgence, à la réémergence de la grande tradition en politique, en désaccord avec la tradition courte aujourd'hui dominante.

Cette contribution impliquera des accommodements, dans une pratique démocratique, entre courants antagonistes, y compris les courants «islamistes» violents qui, comme la plupart des extrémismes, ne perdurent souvent que grâce à la répression physique ciblée de la part d'États très autoritaires, sans légitimité réelle, corrompus et peu mobilisateurs. C'est une pensée musulmane active, voire activiste non violente, pas la pensée répétitive qui règne officiellement. Avec la même passion que celle d'un Qutb et les mêmes risques, elle explicite et accentue, sur la base de la grande tradition, la séparation entre le religieux et le politique. Elle élabore, sur la base de l' authentique sharia, la pleine liberté de conscience et de religion (contre les peines pour conversion à une autre religion ou à l'athéisme déclaré, contre toute forme de la traditionnelle «dhimmitude» et contre toute forme d'anathème pénal contre des personnes), l'égalité entière entre les personnes des deux sexes (contre tous les codes actuels de la famille supposés «coraniques»), le respect de la vie et de l'intégrité personnelle (contre toutes peines de mort et d'amputation, auxquelles le Coran lui-même n'oblige jamais comme unique peine d'un crime ou délit). Même les Frères musulmans historiques, et le Qutb de la Social Justice in Islam (W. Shepard, 1996 (1945)), envisageaient une longue réflexion juridique dans une société déjà réislamisée avant la codification et l'application de ce qu'on appelle couramment, sans pouvoir la définir, la «sharia «, mais l'expérience récente, au Soudan par exemple, montre que c'est hors de la mouvance des Frères musulmans comme, aussi bien, en général, hors de «l'appareil» de l'islam mondial, que la force de mobilisation de l'islamisme ou «islam parallèle» lui-même pourra être, non pas dilapidée ni contrée de front et en bloc, mais réorientée, si la chose est possible. Une vraie Islamic Reformation serait là en germe (B. Beedham, 1994 cité par A.S. Abdin, 1997), disons une Post-Islamist Islamic Self Assertion.

En effet ceux que l'on peut nommer les modernistes non radicaux sont devenus aujourd' hui d'accord avec les fondamentalistes sur l'importance de l'identité islamique (Islamic selfassertion) et des normes musulmanes, même s'ils ont des interprétations divergentes concernant les rapports entre le religieux et le politique. Il restera l'influence posthume (Post-Islamist) de ce que l'on peut estimer être l'épisode ou la parenthèse islamiste. Cette Post-Islamist Islamic self-assertion contient une variété de positions «moyennes», de compromis, de transactions. Ainsi, de fait, la Turquie d'après 1920 passe progressivement et régulièrement d'une laïcité dure aux positions moyennes du modernisme islamique et du néo-traditionalisme islamique, avec lesquelles transige un sécularisme modéré, bref à une réislamisation moderne postislamiste. A l'inverse, mais avec le même aboutissement peut-être, l'Iran d'après 1979 évolue, en fait assez rapidement, d'un radicalisme islamique, non extrémiste toutefois (W. Shepard, 1987; O. Roy, 1992) comme en Afghanistan avec les Taliban ou en Algérie avec les Groupes islamiques armés, aux mêmes positions post-islamistes à peu près qu'en Turquie, à terme (O. Carré, 1993a : 125; O. Roy, 1999 qui adopte le mot «post-islamisme»). Nous sommes aujourd'hui assez loin de cette convergence, mais voilà deux projections idéales qui représentent pour ainsi dire des types. 


\section{CONCLUSION}

Une hypothèse, que je partage puisque j'estime que la fièvre «islamiste» est un épisode, une parenthèse (O. Carré, 1993b : 177 sq.), consiste donc en ce que les quelques États musulmans qui, depuis quelques années, sont sur le modèle de l'Iran, depuis 1979, ou de l'Arabie Saoudite, depuis sa fondation, dirigés par des pouvoirs «islamistes» (Soudan, Afghanistan, et, avec des restrictions, Libye, Pakistan, Mauritanie) suivront la même évolution que le «type» iranien que j'ai évoqué. Le cas saoudien semble plus inflexible mais n'en connaît pas moins, lui aussi, une évolution entre les différentes tendances des élites religieuses et intellectuelles saoudiennes ou résidant en Arabie Saoudite (R. Schulze, 1990 : 395) et son évolution pèse beaucoup sur celle de l'Islam mondial. Une autre hypothèse, à l'inverse, envisage la propagation irrésistible de l'extrémisme islamique, et l'évolution turque, afghane, algérienne, égyptienne, caucasienne, la confirme. L'extrémisme a en effet l'avantage de proposer la réitération aujourd'hui de la ferveur du Prophète lui-même et de la communauté médinoise primitive. De tels «conventicules piétistes» actifs, en effet, peuvent, par la conviction prophétique, «faire éclater les normes religieuses stéréotypées» et produire de «profondes révolutions» économiques, en adaptant non pas les normes existantes mais, à leur racine, «la disposition intérieure sacrée» elle-même, avec une souveraine liberté, selon les mots de M. Weber (1971 :579sq.), comme je l'ai indiqué naguère (O. Carré, 1991 : 17-32 (1986)). Dans l'Iran de la révolution islamique, cet effet mobilisateur est avéré d'une manière générale concernant les institutions (M. Watt, 1988) et quelques réussites socio-économiques précises, dans le cadre, notamment, des services du «Jihâd pour la reconstruction» (J.-P. Digard, 1989; P. Hourcade, 1989). Ces réussites sont tout autre chose que les opérations financières dites «pyramidales» qui ont existé également, sous couvert de l'économie islamique, en Égypte et en Albanie. Il faudrait pouvoir garder ce dynamisme revivaliste et ses fruits positifs bien réels, en en redressant les doctrines et pratiques erronées. Ce doit être possible.

Il est sûr également qu'il y a, pourrait-on dire, plusieurs islams, de par les cultures, les groupes sociaux, les tendances doctrinales ancestrales comme quelques spécialistes de chaque aire musulmane rassemblés l'ont naguère souligné de manière surtout descriptive et orientée principalement sur les aires non arabes, qui sont de loin les plus denses (O. Carré, dir., 1982). Je n'ai ici abordé que les grandes divergences doctrinales et la grande tradition qui les domine nettement toutes. Cette tradition dominante est aussi homogène à travers toutes les cultures, les continents et les siècles, sinon davantage, que l'est celle du judaïsme et du christianisme. Cette grande tradition, sunnite comme shiite, redisons-le, sanctionne et théorise doctrinalement la séparation entre les pouvoirs politiques et la religion, contrairement à la «fusion» dont parlait R. Rémond que je citais au début; elle sanctionne et théorise aussi un quiétisme politique à l'intérieur et à l'extérieur, à l'encontre de l'actuel épisode islamiste violent. Ma perspective ici était plus théologique et islamologique que sociologique, perspective qui n'est pas celle de notre colloque, mais que je crois utile en préambule et comme garde-fous.

\section{BIBLIOGRAPHIE}

ABDIN, A.Sh., 1997, «Modernist interpretation of the status of non-Muslims in Muslim society", The Maghreb Review, vol. 22, n 3-4, London.

ABU ZAYD, N.H., 1999 (1993), Critique du discours religieux : Essais traduits de l'arabe, Sindbad/Actes Sud. 
AKHTAR, S., 1991, The Final Imperative, London, Bellew Publishing Company.

ANAWATI, G., GARDET, L., 1948, Introduction à la théologie musulmane, Paris, Vrin.

ARJOMAND, S.A., 1984, The Shadow of God and the Hidden Imâm : Religion, Political Order and Social Change in Shiite Iran from the begining to 1890, London-Chicago, Chicago University Press.

ARKOUN, M., 1984, Critique de la raison islamique, Paris, Maisonneuve \& Larose. 1986, L'Islam : Morale et Politique(dir.), Paris, DDB.

ASHMAWY, M.S. Al-, 1989, L'islamisme contre l'Islam, Paris \& Caire, Découverte / Fikr (trad.).

AYUBI, N., 1991, Political Islam : Religion and Politics in the Arab World, London, New York, Routledge.

AZMEH, A.AL-, 1993, Islams and modernities, Londres, New York, Verso.

BADRI, Cheikh Yûsûf al-, 1997, Interview à , «Metropolis», 7 juin.

BEEDHAM, B., 1994, «Islamic Reformation», The Economist, August 6th : 14-16.

BLACHERE, R., 1949 \& 1950, Le Coran : Traduction selon un essai de reclassement des sourates, vol. $2 \& 3$, Paris, Maisonneuve.

CALVEZ, J.Y., 1985, La Politique et Dieu, Paris, Cerf.

CAMPICHE, R.J., 1995, Quand les sectes affolent, Genève, Lausanne, Labor \& Fides \& Institut d'Études Sociales.

CARRÉ, O., 1984, Mystique et Politique : Lecture révolutionnaire du Coran par Sayyid Qutb, Frère musulman radical, Paris, Cerf \& Presses FNSP.

, 1986, «A propos de Weber et l'Islam», Archives de sciences sociales des religions, $61 / 1: 139-152$.

1991, L'utopie islamique dans l'Orient arabe, Paris, Presses FNSP.

1993a, L'Islam lä̈que ou le retour à la grande tradition, Paris, A. Colin.

1993b, Le nationalisme arabe, Paris, Fayard.

dir., 1982, L'Islam et l'État dans le monde d'aujourd'hui, Paris, P.U.F. Trad. anglaise : 1987, New-Delhi, Manohar.

\& MICHAUD, G., 1983, Les Frères musulmans : Égypte, Syrie 1928-1982, Gallimard, Paris 1983.

COURBAGE, Y., FARGUES, P., 1992, Chrétiens et Juifs dans l'Islam arabe et turc, Paris, Fayard.

CRONE, P., HINDS, M., 1986, God's Caliph : Religious Authority in the First Centuries of Islam, Cambridge.

1983, «Déclaration islamique universelle des droits de l'homme», Islamochristiana $\mathrm{n}^{\circ} 9$ (Rome) : p.1-19 et 103-140.(trad. littérale annotée). 
DIGARD, J.-P., \& KARIMI, A., 1989, «Les Baxtyari sous influence occidentale :Acculturation et déculturation» in RICHARD(Y).

Hicham, DJAIT, 1989, La grande discorde : Religion et politique dans l'Islam des origines, Paris, Gallimard.

1979, «Dustûr al-islâmî (al-)» «La Constitution islamique», Majallat al-Azhar, Caire, Avril.

HOURCADE, P., 1989, "Vaqf et modernité en Iran : Les agro-business de l'Astân-e qods de Mashad», in RICHARD, Y.

IMARA, M., 1978, Al-islâm wa-l-sulta al-dîniyya (Islam et pouvoir religieux), Le Caire.

JADD AL-HAQQ, A., 1982, «Réplique à l'obligation manquante» (en arabe), Al-Ahrâm 8.01.

LAMBTON, A.K., 1981, State and Government in Medieval Islam : The Jurists, Oxford, NewYork, Oxford University Press.

LAOUST, H., 1939, Essai sur les doctrines sociales et politiques de Takî al-Dîn Ahmad b. Taïmîya, Cairo, IFAO.

1965 (1977), Les schismes dans l'islam: Introduction à une étude de la religion musulmane, Paris, Payot.

LAROUI, A., 1987, Islam et modernité, Paris, Découverte.

LEWIS, B., 1988, Le langage politique de l'Islam, Paris, Gallimard.

MAHFOUZ, N., 1995 (1958), Les fils de la médina (trad. J.-P. Guillaume), Sindbad/Actes Sud, $2 \mathrm{e}$ éd., épuisé.

MANSOUR, C., 1975, L'autorité dans la pensée musulmane : le concept d'ijmâ, consensus, et la problématique de l'autorité, Paris, Vrin.

MAYER, A.E., 1991, Islam and Human rights, Boulder, Westview Press.

1983, Model of an Islamic Constitution/ Modèle de Constitution islamique (official transl.), Islamic Council/Conseil islamique, Londres, Islamabad.

MORABIA, A., 1993, Le Gihad dans l'Islam médiéval : Le «combat sacré» des origines au XII siècle, Paris, A. Michel 1993.

NA'IM, A. An-, 1990, Towards an Islamic Reformation, New York, Syracuse University Press.

QUTB, Sayyid, 1996 (1949), Social Justice in Islam, Voir SHEPARD, W.E., 1996.

1979 (1952-1965, vol. 1-30), In the shade of the Qur'ân, vol. 30, transl. Salahi (A.) \& Shamis (A.A), London.

REMOND, R., 1999, France culture, «Les lundis de l'histoire» du 18.01.99 à propos de : 1998, Religion et société en Europe: Essai sur la sécularisation des sociétés européennes aux XIX ${ }^{e}-X X^{e}$ siècles (1789-1998), Paris, Seuil.

RICHARD, Y., 1989, Entre l'Iran et l'Occident: Adaptation et assimilation des idées et techniques occidentales en Iran, Paris, Ed. de la Maison des sciences de l'homme.

ROY, O., 1992, L'échec de l'Islam politique, Paris, Seuil. 
,1999, «Le post-islamisme», Revue du Monde musulman et de la Méditerranée 85 86, Edisud.

SHEPARD, W.E., 1987, «Islam and Ideology : towards a Typology», International Journal of Middle East Studies 19:307-336.

1996, Sayyid Qutb and Islamic Activism : A translation and Critical Analysis of "Social Justice in Islam», Leiden, London, E.J. Brill.

SCHULZE, Reinhard, 1990, Islamischer Internationalismus im 20. Jahrhundert: Untersuchungen zur Geschichte der Islamischen Weltliga, Leiden, E.J. Brill 1990.

SINNO, A., 1995, «La pensée de Nasr Hamîd Abû Zayd», Centre d'étude du Monde arabe moderne (CEMAM), Lettre du 22.12.

SIVAN, E., 1985 (1991), Radical Islam : Mediaeval Theology and Modern Politics, New Haven.

TAHA, M.M., 1987, The second message of Islam, transl. Na'îm (A.An-), Syracuse, N.Y.

1981 : Universal Islamic Declaration of Human rights/Déclaration islamique universelle des droits de l'homme (official transl.), Islamic Council for Europe /Conseil Islamique de l'Europe, Paris, Londres.

VATIKIOTIS, P.J., 1992 (1987), L'Islam et l'État, trad., Paris, Gallimard.

WATT, W.M., 1988, Islamic Fundamentalism and Modernity, Londres, Routledge.

WEBER, M., 1971, Economie et société, trad., Paris, Plon. 